\title{
Structural MRI Correlates of Episodic Memory Processes in Parkinson's Disease Without Mild Cognitive Impairment
}

\author{
Eva Pirogovsky-Turk ${ }^{\mathrm{a}, \mathrm{b}}$, J. Vincent Filoteo ${ }^{\mathrm{a}, \mathrm{b}, \mathrm{c}}$, Irene Litvan ${ }^{\mathrm{d}}$ and Deborah L. Harrington ${ }^{\mathrm{b}, \mathrm{e}, *}$ \\ ${ }^{a}$ Department of Psychiatry, University of California San Diego, USA \\ ${ }^{\mathrm{b}}$ Research Service, VA San Diego Healthcare System, USA \\ ${ }^{\mathrm{c}}$ Psychology Service, VA San Diego Healthcare System, USA \\ ${ }^{\mathrm{d}}$ Department of Neurosciences, University of California San Diego, USA \\ ${ }^{\mathrm{e}}$ Department of Radiology, University of California San Diego, USA
}

\begin{abstract}
.
Background: Changes in episodic memory are common early in Parkinson's disease (PD) and may be a risk factor for future cognitive decline. Although medial temporal lobe (MTL) memory and frontostriatal (FS) executive systems are thought to play different roles in distinct components of episodic memory impairment in PD, no study has investigated whether different aspects of memory functioning are differentially associated with MTL and FS volumes in nondemented patients without mild cognitive impairment (PD-woMCI).

Objectives: The present study investigated MRI markers of different facets of memory functioning in 48 PD-woMCI patients and 42 controls.

Methods: Regional volumes were measured in structures comprising the MTL and FS systems and then correlated with key indices of memory from the California Verbal Learning Test.

Results: In PD-woMCI patients, memory was impaired only for verbal learning, which was not associated with executive, attention/working memory, or visuospatial functioning. Despite an absence of cortical atrophy, smaller right MTL volumes in patients were associated with poorer verbal learning, long delayed free recall, long delayed cued recall, and recognition memory hits and false positives. Smaller right pars triangularis (inferior frontal) volumes were also associated with poorer long delayed cued recall and recognition memory hits. These relationships were not found in controls.

Conclusions: The findings indicate that MTL volumes are sensitive to subtle changes in almost all facets of memory in PD-woMCI, whereas FS volumes are sensitive only to memory performances in cued-testing formats.

Keywords: Parkinson's disease, magnetic resonance imaging, memory, neuropsychology, medial temporal lobe system, frontostriatal system, cognitive functions
\end{abstract}

\section{INTRODUCTION}

Cognitive impairments are found early in the course of Parkinson's disease (PD), and up to $80 \%$ of patients show dementia 8-years later [1]. Dementia lowers the quality of life for patients and caregivers alike, and

\footnotetext{
*Correspondence to: Deborah L. Harrington, Ph.D., VA San Diego Healthcare System 116A-13, 3350 La Jolla Village Drive, San Diego CA 92161, USA. Tel.: +1 858-552-8585/Ext. 7851; E-mail: dharrington@ucsd.edu.
}

portends a greater risk of nursing home placement and mortality [2, 3]. Markers of cognitive functioning in PD are needed that predate the onset of mild cognitive impairment (MCI). Once markers are identified, they can then be studied longitudinally to chart the rate of disease progression and determine which markers predict risk for dementia.

Episodic memory impairment is common early in PD and may be a risk factor for future cognitive decline $[4,5]$. However, neuroanatomical substrates of subtle 
changes in memory that predate cognitive impairment have not been comprehensively studied in nondemented PD patients without mild cognitive impairment (PD-woMCI) using recent criteria [6]. Most investigations have almost solely focused on the relationship of memory to hippocampus and entorhinal cortex morphometry, owing to their roles in memory encoding and consolidation. Cross-sectional studies of combined samples of PD patients with and without cognitive impairment typically report that medial temporal lobe (MTL) volumes correlate with various aspects of verbal memory including delayed recall and recognition memory [7-13]. However, frontostriatal (FS) networks also mediate executive aspects of episodic memory, which govern attention and working memory and selfinitiated search and monitoring strategies at encoding and retrieval $[14,15]$. Recently, our group found that poorer long delayed free recall in nondemented PD was associated with reduced MTL and FS volumes in both hemispheres, suggesting that memory and executive systems are associated with early, subtle memory changes [16]. This study may have included some patients with MCI, thereby preventing an examination of the systems associated with memory functioning before mild cognitive impairment [6].

In clinical evaluations of PD, different components of verbal memory are typically examined including learning, retention, retrieval, and the types of errors during recall. It is traditionally believed that impaired learning in nondemented PD is largely due to inefficient use of strategies during encoding because of FS dysfunction [17]. For example, one study reported that poorer verbal learning in PD patients with and without MCI was not related to hippocampal atrophy [7], speculating that verbal encoding deficits, secondary to inefficient use of learning strategies, may be related to changes in the FS system, which was not examined. To our knowledge, no study has directly tested this hypothesis by correlating multiple facets of verbal learning and memory with FS volumes.

Another important distinction is whether a patient has retention or retrieval difficulties. It is widely believed that PD patients exhibit retrieval deficits. Indeed, nondemented PD patients demonstrate impaired free recall, yet memory performance can improve to normal or near normal levels when cues or yes/no recognition testing formats are used [18-20], which decrease retrieval demands. This memory profile is attributed to deficient retrieval rather than retention of stored information, ostensibly due to FS dysfunction, which disrupts strategies for search and retrieval of information [18]. In contrast, patients with
MTL damage (e.g., Alzheimer's disease) show rapid rates of forgetting and fail to benefit from cueing and recognition testing, owing to impaired consolidation secondary to hippocampal atrophy $[18,21]$.

However, nondemented PD patients can present with different patterns of memory impairment [22-26]. Our group found retrieval deficits (i.e., significantly better recognition memory than free recall) in about $1 / 3$ of PD patients [23]. The remaining patients did not benefit from recognition memory testing due to increased endorsement of non-list items as targets (false positives), presumably related to impaired inhibition secondary to FS dysfunction. It is also possible that lack of benefit from recognition memory testing in PD was related to impaired encoding/consolidation due to hippocampal dysfunction. This demonstrates the importance of assessing multiple components of memory. Moreover, while assumptions are frequently made in clinical practice about the different roles of FS and MTL systems in distinct components of episodic memory impairment, no study has investigated whether various aspects of memory functioning are differentially associated with MTL and FS volumes in PD-woMCI. This is an important question because neuroanatomical correlates of different facets of memory may suggest MRI markers of future memory decline, which could then be studied longitudinally to determine if they are risk factors for dementia.

The current study sought to elucidate MRI markers of subtle changes in different aspects of episodic verbal memory in PD-woMCI, which was evaluated by a widely used test (California Verbal Learning Test II- Short Form) [27]. We focused on measures that are routinely used in clinical assessments of PD [27] and are theoretically relevant, namely indices of learning, long delay free recall, retention, cued retrieval (cued recall relative to free recall), and recognition retrieval (recognition memory relative to free recall). These measures were correlated with regional volumes comprising the MTL memory and the FS executive systems. We hypothesized that poorer verbal learning would most strongly correlate with FS volumes, secondary to difficulties in executive aspects of encoding. We also hypothesized that the relative improvement in delayed recall with cued recall and recognition memory formats (cued retrieval and recognition retrieval indices) would best correlate with FS volumes, since these indices emphasize executive aspects of retrieval. In contrast, long delay free recall and retention were predicted to correlate most strongly with MTL volumes, owing to their emphasis on memory consolidation. 


\section{METHOD}

\section{Participants}

The University of California San Diego Human Research Protections Program approved this study. Study participants included 48 PD-woMCI (see definition for MCI below) and 42 healthy controls (HC) who were spouses of patients or community volunteers. Participants provided written informed consent prior to study procedures. Thirty-eight of the HC participants and 43 of the PD participants partially overlapped with the cohort from our prior study that investigated associations between individual differences in volumes of four brain systems and performances in five cognitive domains [16]. Exclusion criteria included metal in the head, neurological diagnoses other than PD, psychiatric diagnoses, MCI, history of alcohol or substance abuse, positive MRI findings (e.g., infarcts, clinically significant vascular disease), and use of anticholinergics or cognitive medications (e.g., donepezil). PD participants met the PD United Kingdom Brain Bank Criteria. All PD patients were taking levodopa or levodopa combination therapy and were tested while on medication. The groups did not differ in gender composition, years of education, age, or Mini-Mental Status Exam (MMSE) scores (Table 1). Motor symptoms were assessed using Part III of the Unified Parkinson's Disease Rating Scale (UPDRS) and the Hoehn and Yahr scale. Motor symptom severity and disease duration (Table 1) were similar to other PD-woMCI samples [28-31].

\section{PD-woMCI criteria}

PD participants were excluded if they met criteria for PD-MCI based on a modified (no language test) Level 1 Movement Disorders Society task force criteria [6], which was assessed using four cognitive domains: 1) Executive function: Letter Fluency (number correct) from the Delis Kaplan Executive Function System (DKEFS), 2) Verbal memory: CLVT long delayed free recall (total correct), 3) Attention/working memory: Digit Span Backward (Wechsler Adult Intelligence Scale - III; total correct), and 4) Visuospatial function: Benton Judgment of Line Orientation test (JLOT; version $\mathrm{V}$, number correct out of 15 items). Although we did not have a measure of language, as recommended for a Level 1 PD-MCI diagnosis, language skills are typically spared in patients without deficits in other cognitive domains [28]. Owing to the high educational level of our sample, raw scores for the cognitive tests were transformed to $\mathrm{z}$-scores based on the $\mathrm{HC}$ group mean and standard deviation [6]. PD-MCI was diagnosed if a patient demonstrated a z-score $\leq-1.5$ on at least 2 neuropsychological measures. Of 56 patients in the original sample, 48 met criteria for PDwoMCI. There were no significant differences between $\mathrm{PD}$-woMCI and $\mathrm{HC}$ groups in executive functioning, attention/working memory, visuospatial function, or verbal memory (long delay free recall) (Tables 1 and 2).

\section{Memory assessment}

The California Verbal Learning Test - Second Edition Short Form (CVLT) [27] was used to assess memory. CVLT variables were first normalized to the $\mathrm{HC}$ group by calculating $\mathrm{z}$-scores based on the $\mathrm{HC}$ group mean/standard deviation. We then calculated the following CVLT indices using the z-scores: 1) learning [number of words recalled on learning trials 1-4], 2) long delay free recall [number of words recalled on the long delay free recall trial], 3) retention [number of words recalled on trial 4 - number of words on long delay free recall], 4) cued retrieval [cued recall d prime (i.e., a discriminability index) [27] - long delay free recall] and 5) recognition retrieval [recognition d prime - long delay free recall] [23, 32]. Analyses were also conducted for sub-measures of the retrieval indices. Sub-measures comprising the cued retrieval index included long delay cued recall [number of target words recalled] and cued recall intrusions [number of non-list words recalled]. Sub-measures comprising the recognition retrieval index included recognition memory hits [number of target words recognized] and false positives [number of non-list items endorsed]. Higher values on learning, long delayed free recall, long delay cued recall, and hits indicate better performance. Higher values on retention, cued retrieval, recognition retrieval, false positives, and cued recall intrusions indicate worse performance.

\section{MRI procedures}

High resolution T1-weighted anatomic images were acquired on a GE 3T Excite MRI system. Automated reconstruction and volumetric segmentation of images were conducted using FreeSurfer 5.1 (http://surfer.nmr.mgh.harvard.edu/), which is widely used and provides accurate renderings of regional volumes without rater bias. We used an imaging protocol that maximizes differentiation of the white and gray matter boundary (3D spoiled gradient-recalled at steady state, TE $3.0 \mathrm{~ms}$, TR $7.8 \mathrm{~ms}$, TI $600,8^{\circ}$ flip angle, NEX 1, 1-mm axial slice thickness, FOV 
Table 1

Demographics, disease characteristics, and neuropsychological test performances of participants

\begin{tabular}{|c|c|c|c|c|}
\hline & Parkinson's $(n=48)$ & Controls $(n=42)$ & $\mathrm{F}$ & $p$ \\
\hline \multicolumn{5}{|l|}{ Demographics } \\
\hline Age (years) & $66.9(7.9)$ & $66.7(8.3)$ & 2.5 & 0.12 \\
\hline Education (years) & $17.2(3.0)$ & $17.1(2.8)$ & 0.04 & 0.85 \\
\hline Gender $(\mathrm{M} / \mathrm{F})^{\mathrm{a}}$ & $25 / 23$ & $21 / 21$ & 0.39 & 0.84 \\
\hline Mini-Mental Status Exam & $29.0(1.4)$ & $29.1(0.9)$ & 2.3 & 0.14 \\
\hline \multicolumn{5}{|l|}{ Disease Characteristics } \\
\hline Duration of PD (years) & $6.2(4.6)$ & & & \\
\hline UPDRS III & $27.8(10.0)$ & & & \\
\hline \multicolumn{5}{|l|}{ Hoehn \& Yahr stage } \\
\hline Stage 1/2/2.5/3 (frequency) & $3 / 15 / 17 / 13$ & & & \\
\hline Levodopa dosage equivalence & $678.7(570.0)$ & & & \\
\hline \multicolumn{5}{|l|}{ Neuropsychological Tests } \\
\hline Executive: Letter Fluency & $44.5(10.6)$ & $48.1(11.5)$ & 2.4 & 0.13 \\
\hline $\begin{array}{l}\text { Attention/Working Memory: } \\
\text { Digit Span Backward }\end{array}$ & $7.4(1.8)$ & $7.8(2.3)$ & 0.63 & 0.43 \\
\hline Visuospatial: JLOT & $12.0(2.1)$ & $12.7(1.9)$ & 2.3 & 0.13 \\
\hline \multicolumn{5}{|l|}{ Brain Volumes $^{\mathrm{b}}$} \\
\hline R Pars triangularis & $0.27(0.05)$ & $0.27(0.05)$ & 0.03 & 0.86 \\
\hline R Entorhinal & $0.12(0.03)$ & $0.13(0.02)$ & 1.2 & 0.28 \\
\hline R Parahippocampus & $0.14(0.02)$ & $0.14(0.02)$ & 1.2 & 0.44 \\
\hline R Hippocampus & $0.25(0.03)$ & $0.26(0.03)$ & 0.60 & 0.28 \\
\hline L Temporal Pole & $0.16(0.03)$ & $0.16(0.03)$ & 0.38 & 0.54 \\
\hline
\end{tabular}

Demographics, disease characteristics, and neuropsychological test performances are presented as the mean (standard deviation) of raw scores. Brain volumes for regions that significantly correlated with memory variables in the Parkinson's group are presented. Frequency counts are presented for gender and the Hoehn and Yahr stages. ${ }^{\text {a }}$ Chi-square test for group differences. Test statistic in the F column is a chi-square value. ${ }^{b}$ Brain volumes $\left(\mathrm{mm}^{3} / \mathrm{ICV} \mathrm{m^{3 }}\right)$ for regions that were significantly correlated with various CVLT measures. UPDRS $=$ Unified Parkinson's Disease Rating Scale; JLOT = Judgment of Line Orientation; R = right hemisphere; L = left hemisphere.

Table 2

Performances on the verbal memory measure (CVLT)

\begin{tabular}{|c|c|c|c|c|c|c|}
\hline \multirow[b]{2}{*}{ CVLT Variables } & \multicolumn{2}{|c|}{ Parkinson's } & \multicolumn{4}{|c|}{ Controls } \\
\hline & Mean (SD) & Min, Max & Mean (SD) & Min, Max & $\mathrm{F}$ & $p$ \\
\hline Learning & $27.5(3.5)$ & 19,35 & $29.4(3.1)$ & 23,35 & 7.4 & 0.008 \\
\hline Long Delay Free Recall & $7.0(1.8)$ & 3,9 & $7.5(1.4)$ & 4,9 & 2.4 & 0.13 \\
\hline Retention & $0.8(1.4)$ & $-2.0,4.0$ & $0.6(1.2)$ & $-2.0,3.0$ & 0.16 & 0.69 \\
\hline Cued Retrieval $^{\mathrm{a}}$ & $14(0.8)$ & $-2.2,1.6$ & $0.0(0.6)$ & $-1.3,1.5$ & 0.88 & 0.35 \\
\hline Long Delay Cued Recall & $7.4(1.5)$ & 3,9 & $7.8(1.1)$ & 5,9 & 2.3 & 0.13 \\
\hline Cued Recall Intrusions $^{\mathrm{b}}$ & $0.3(0.6)$ & 0,2 & $0.3(0.7)$ & 0,3 & & 0.95 \\
\hline Recognition Retrieval $^{\mathrm{a}}$ & $0.1(1.1)$ & $-2.9,2.3$ & $0.0(0.9)$ & $-2.2,2.7$ & 0.14 & 0.71 \\
\hline Hits $^{\mathrm{b}}$ & $8.6(0.6)$ & 7,9 & $8.6(0.7)$ & 6,9 & & 0.76 \\
\hline False Positives $^{b}$ & $1.4(1.7)$ & 0,6 & $0.9(1.1)$ & 0,5 & & 0.15 \\
\hline
\end{tabular}

Note: Memory measures are from the California Verbal Learning Test - Short form (CVLT). ${ }^{\mathrm{a} C V L T}$ scores are raw scores, except for cued retrieval and recognition retrieval, which are $\mathrm{z}$-scores, since they involved subtraction of measures using different metrics. ${ }^{\mathrm{b}} \mathrm{Group}$ comparisons used the Mann-Whitney U test due to the skewed distributions of these variables.

$25.6 \mathrm{~cm}, 256 \times 256$ matrix). MRIs for each participant were motion corrected, normalized for intensity in homogeneities, and transformed to Talairach space. Non-brain tissue was removed using a hybrid watershed/surface deformation procedure, subcortical structures were segmented, and further intensity normalization was conducted. This was followed by white-matter segmentation, tessellation of the gray-white matter boundary, and automated topol- ogy correction. Finally, surface deformation following intensity gradients optimally placed the gray/white and gray/cerebrospinal fluid borders at the location where the greatest shift in intensity defines the transition to the other tissue class. The cerebral cortex was parcellated into 34 gyral-based regions in each hemisphere based on the Desikan-Killiany atlas [33]. Subcortical structures were delineated by an algorithm that examines variations in voxel intensities and spatial relationships. 
Anatomical accuracy of the segmentations was manually verified. To account for differences in head size, volumes for each region were divided by intracranial volume (ICV).

\section{Regions of interest}

Morphometric analyses were conducted on a subset of regions that were the focus of our hypotheses (Fig. 1, left panel), namely the FS executive system (frontal cortical regions including caudal middle frontal gyrus, rostral middle frontal gyrus, superior frontal gyrus, areas of the inferior frontal gyrus including pars triangularis, pars opercularis, pars orbitalis, and striatal regions including caudate, putamen) and the MTL memory system (temporal pole, entorhinal cortex, parahippocampus, hippocampus) [16].

\section{Statistical analyses}

Z-scores for memory measures were residualized by calculating the residuals from the regression of age and gender onto each brain volume for each group separately. Since levodopa dosage and other clinical variables (i.e., disease severity, motor symptom severity on the UPDRS Part III) could have an effect on cognition in PD, correlations were performed between CVLT variables and clinical variables. The CVLT variables did not significantly correlate with the UPDRS or disease duration. $(p>0.05)$. However, CVLT learning $(r=-.33, p=0.011)$ and retention $(r=-.31, p=0.02)$ significantly correlated with levodopa dosage equivalence, indicating that higher levodopa dosage equivalences were associated with poorer learning and retention. Therefore, residuals from the regression of levodopa equivalencies, age,
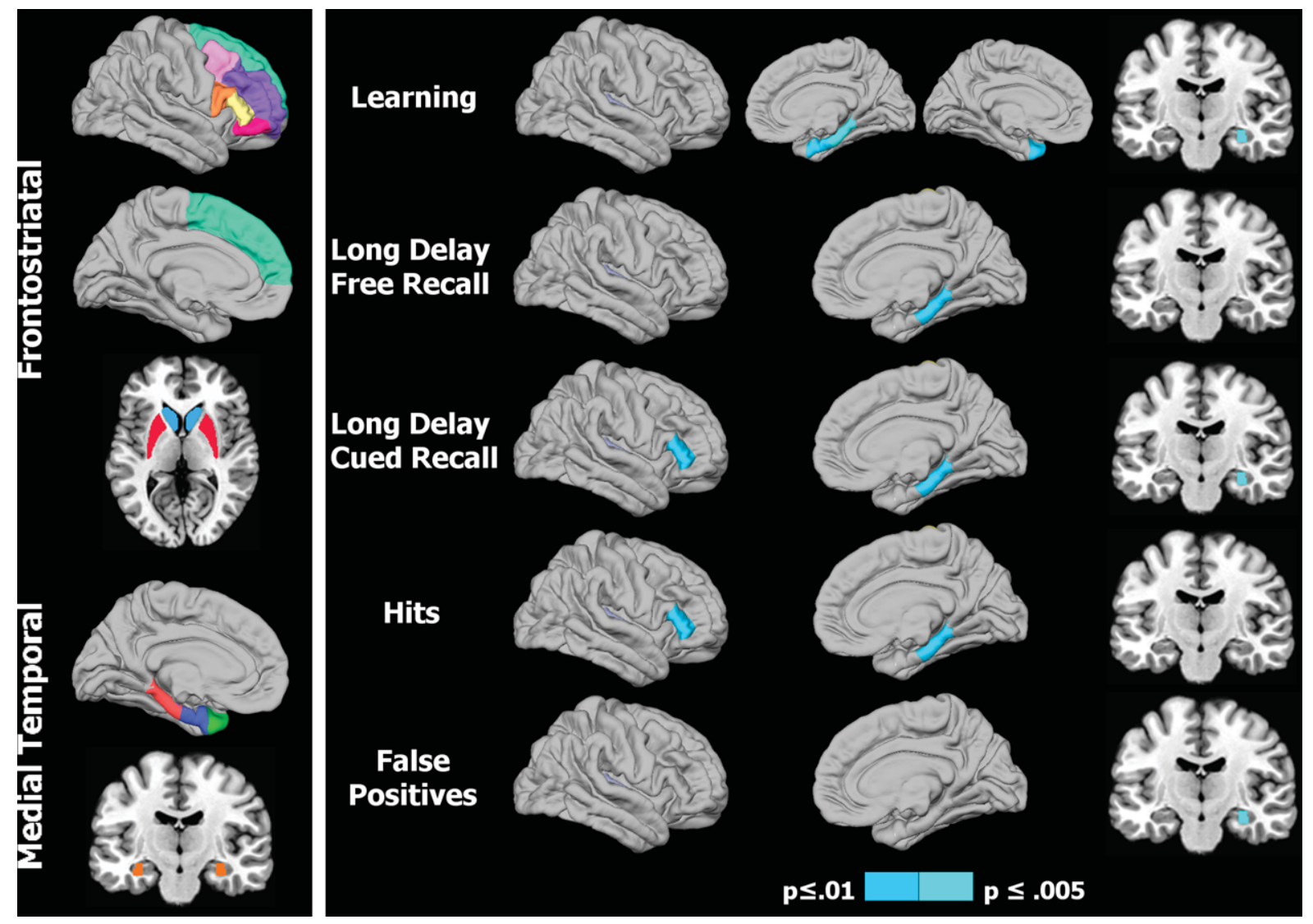

Fig. 1. Regional volumes of interest and their association with memory in Parkinson's patients. 1) Left panel: Colored areas designate regions of interest (ROI) in the frontostriatal and medial temporal systems. ROIs on sagittal surfaces are displayed for one hemisphere, but were analyzed for homologous areas of both hemispheres. The caudate/putamen and the hippocampus are respectively shown on axial and coronal views. 2) Right panel: Right hemisphere cortical (lateral/medial sagittal surfaces) and hippocampus (coronal view) volumes that significantly correlated with various memory measures. For CVLT learning (top row), the left hemisphere medial surface displays the significant correlation with the temporal pole. The $p$ value for correlation coefficients is designated by the color bar; $p \leq 0.01$ (dark blue) and $p \leq 0.005$ (light blue). 
and gender onto CVLT learning and CVLT retention were calculated for each group separately. For all other CVLT variables, residuals from the regression of age and gender onto these CVLT variables were calculated for each group separately. For most variables, Pearson correlations were conducted between the residuals for the CVLT variables and regional volumes, separately for each group. Spearman rank correlations were conducted for cued recall intrusions and recognition memory hits and false positives due to skewed distributions of measures. Owing to our a priori hypotheses, which were driven by previous results [16], a more stringent uncorrected statistical threshold of $p \leq 0.01$ was adopted in lieu of overly conservative adjustments [34]. This threshold was chosen in an attempt to strike a balance between Type I and Type II errors. Nonetheless, the results should be interpreted with caution.

\section{RESULTS}

\section{Memory performance}

T tests and Mann-Whitney U tests (cued recall intrusions, recognition memory hits and false positives) compared the groups on the CVLT variables (Table 2). The PD-woMCI group performed significantly worse than the HC group on learning $(p=0.008)$. A repeatedmeasure ANOVA showed that patients' rate of learning was similar to that of the HC group across all 4 learning trials (nonsignificant group $\mathrm{X}$ trial interaction; mean (SD) for the slope of the learning function was 0.80 (.43) and 0.78 (.38) for the PD-woMCI and HC groups, respectively). Thus, verbal learning was poorer in the PD-woMCI group, irrespective of the learning trial. In patients, learning was not correlated $(p>0.20)$ with measures of executive functioning, attention, or visuospatial functioning (Table 1). Partial correlations, adjusting for levodopa dosage equivalence, also showed no significant associations between learning and measures of executive functioning, attention, or visuospatial functioning. Group differences were not found on any other memory measures. Importantly, the range of values was more restricted for retention, cued retrieval, cued recall intrusions, and recognition retrieval relative to all other measures. Additionally, a cued retrieval-deficit profile ( $\mathrm{z}$-score $>1.5$ difference between cued recall $\mathrm{d}$ prime and long delay free recall) was found in only $8 \%(n=4)$ of PD-woMCI and $2 \%$ $(n=1)$ controls. A recognition retrieval-deficit profile ( $\mathrm{z}$-score $>1.5$ difference between recognition memory $\mathrm{d}$ prime and long delay free recall) was found in $14.5 \%$ $(n=7)$ of PD-woMCI and 5\% $(n=2)$ of controls.

\section{Group differences in regional volumes}

Group comparisons ( $t$-tests) showed right putamen volume loss (HC: $M=0.35, S D=0.05$; PD-woMCI: $M=0.33, S D=0.04 ; p=0.005)$ and a nonsignificant trend for left putamen volume loss (HC: $M=0.36$, $S D=0.06$; PD-woMCI: $M=0.34, S D=0.04 ; p=0.02$ ) in the PD-woMCI group. No other group differences were found in MTL/FS volumes.

\section{Correlation of regional volumes with different components of memory}

Figure 1 (right panel) and Table 3 display the regional maps and significant correlation coefficients between brain volumes and performances on CVLT

Table 3

Significant correlations between regional frontostriatal and medial temporal lobe volumes and episodic verbal memory performance in the Parkinson's group

\begin{tabular}{lcccc}
\hline Regions & Learning & $\begin{array}{c}\text { Long Delay } \\
\text { Free Recall }\end{array}$ & $\begin{array}{c}\text { Long Delay } \\
\text { Cued } \\
\text { Recall }\end{array}$ & $\begin{array}{c}\text { Recognition } \\
\text { Memory } \\
\text { Hits }\end{array}$ \\
$\begin{array}{l}\text { Frontostriatal System } \\
\text { R Pars triangularis }\end{array}$ & & & $0.33^{\mathrm{a}}$ & $0.35^{\mathrm{a}}$ \\
Fedial Temporal System & & & & \\
R Entorhinal & $0.39^{\mathrm{a}}$ & & $0.33^{\mathrm{a}}$ & $0.33^{\mathrm{a}}$ \\
R Parahippocampus & $0.44^{\mathrm{b}}$ & $0.34^{\mathrm{a}}$ & $0.39^{\mathrm{b}}$ & \\
R Hippocampus & $0.37^{\mathrm{b}}$ & & & $-0.40^{\mathrm{b}}$ \\
L Temporal Pole & $0.36^{\mathrm{a}}$ & & & \\
\hline
\end{tabular}

Only memory indices that exhibited significant correlations with brain volumes are displayed. Pearson correlation coefficients are reported, except for recognition memory hits and false positives, which are Spearman correlation coefficients. $R=$ right hemisphere; $\mathrm{L}=$ left hemisphere. ${ }^{\mathrm{a}} p \leq 0.01 ;{ }^{\mathrm{b}} p \leq 0.005$. 

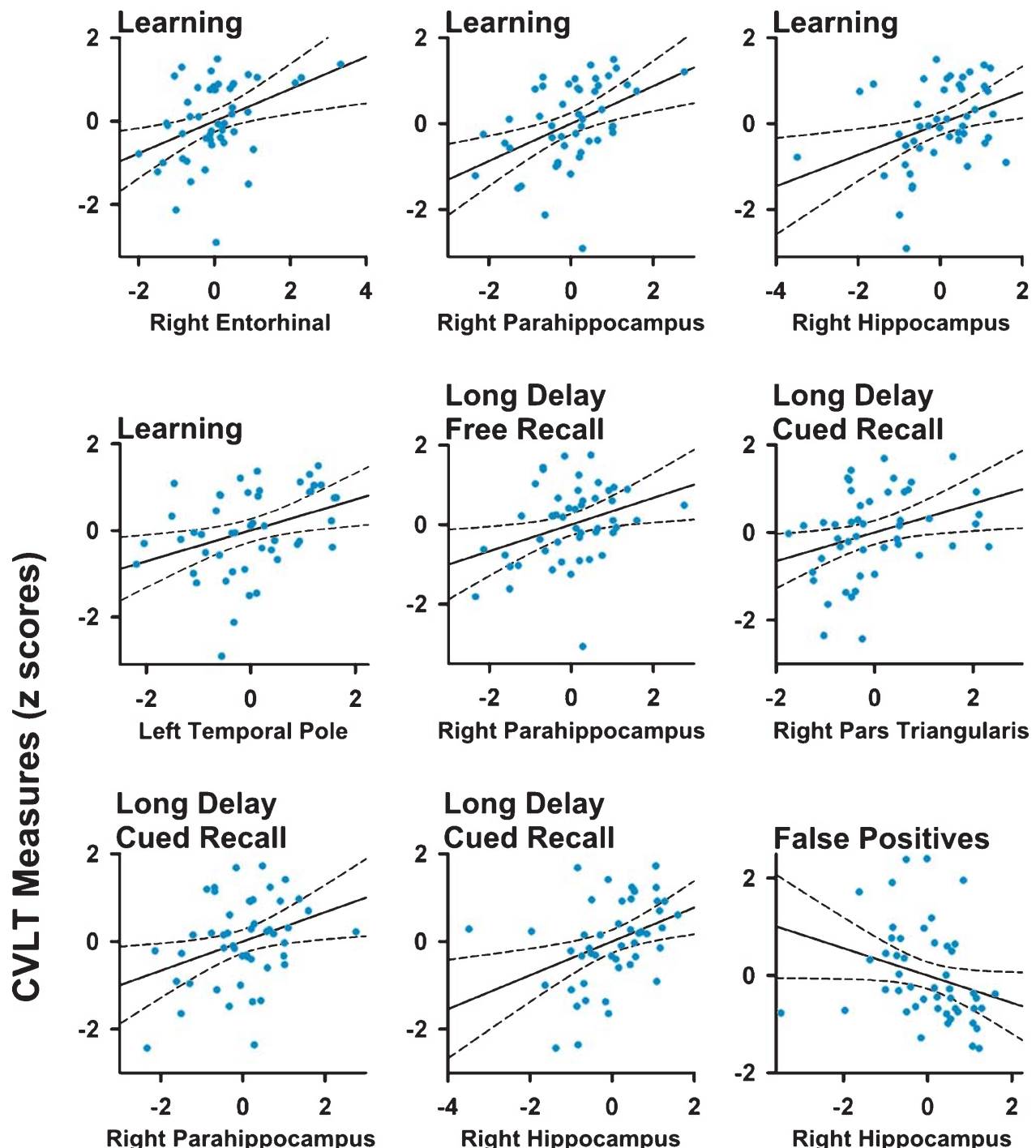

Right Parahippocampus
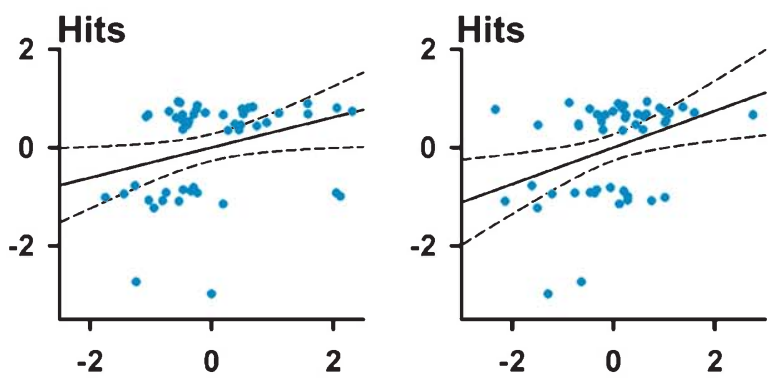

Right Pars Triangularis

Right Parahippocampus

\section{Volumes (z scores)}

Fig. 2. Scatter plots showing significant correlations between measures of memory and regional volumes in Parkinson's patients. For most variables, age and gender adjusted residuals for MRI volumes and CVLT measures are plotted. An exception is CVLT learning, for which levodopa dosage equivalence, age, and gender adjusted residuals are plotted. Solid and dotted lines display the best-fitting linear regression line and $95 \%$ confidence intervals. 
variables for the PD-woMCI group. Figure 2 shows scatter plots of the significant correlations (age and gender adjusted residuals). In the PD-woMCI group, worse learning (trials 1 to 4 ) correlated with smaller right entorhinal, right parahippocampus, right hippocampus, and left temporal pole volumes. Poorer long delayed free recall correlated with smaller right parahippocampus volume. Brain volumes did not correlate with retention or the long delayed cued retrieval and recognition retrieval indices. As for submeasures of cued retrieval, poorer long delayed cued recall correlated with smaller right pars triangularis, parahippocampus, and hippocampus volumes. Cued recall intrusions did not correlate with brain volumes. As for sub-measures of recognition retrieval, greater hits correlated with larger right pars triangularis and hippocampus volumes, whereas more false positives correlated with smaller right hippocampus volumes. No significant correlations were found in the HC group. Brain volumes for regions that significantly correlated with various memory variables are displayed in Table 1.

\section{DISCUSSION}

Our results principally linked subtle changes in different components of memory functioning in PDwoMCI with volumes of the MTL. This was found even for measures expected to emphasize FS functioning, namely learning and recognition memory false positives. Our results are compatible with emerging studies that reveal interactions between dopamine neurotransmission and hippocampal synaptic plasticity in memory [35]. Memory impairments that predate or predict dementia are also associated with a loss of cholinergic function and the accumulation of Lewy Bodies, amyloid plaques, and neurofibrillary tangles $[36,37]$. In contrast, we found that FS volumes were only associated with sub-measures of the cued retrieval and recognition indices, namely long delayed cued recall and recognition memory hits, which correlated with smaller volumes of the right pars triangularis, an element of the right-hemisphere ventral-attention system [38]. This is fitting as the ventral prefrontal cortex modulates reorienting responses to task-relevant stimuli [38-40], which is elicited by cued memory testing formats. These neurocognitive relationships were not found in controls, suggesting that they are related to subtle changes in the disease, rather than normal aging.

Episodic memory in PD-woMCI was significantly worse than controls only for learning, which was not correlated with executive functioning, as it is in cognitively impaired PD [18], nor FS volumes. These findings suggest that poorer learning was not related to executive aspects of encoding, but rather diminished encoding functions of the MTL. Indeed, poorer learning correlated with smaller right-hemisphere hippocampus, parahippocampus, and entorhinal cortex volumes and left-hemisphere temporal pole volume, unlike other studies [7]. This result is consistent with right-hemisphere MTL mediation of verbal learning and integration of contextual information (e.g., recency, novelty, familiarity) for encoding and retrieval [41-43]. Long delayed free recall was also associated with smaller right parahippocampal volume. The finding that verbal learning and memory were associated with mostly right hemisphere, medial temporal lobe volumes was somewhat surprising, but is consistent with a study reporting an association between right, but not left hippocampal volumes and verbal learning in PD patients without dementia [7]. Future studies in larger samples of PD-woMCI should be conducted to further examine this intriguing finding. Previously we reported associations between long delay free recall and bilateral MTL (right and left hippocampus and entorhinal cortex; left temporal pole) and bilateral FS (right and left pars triangularis and pars orbitalis, left superior frontal, right putamen) volumes in nondemented PD [16]. More pervasive bilateral hemispheric associations may have been driven by the inclusion of PD-MCI patients, which underscores the importance of characterizing MCI status when studying neurocognitive relationships.

As for other primary memory indices, recognition retrieval, cued retrieval, and retention were also not impaired in PD-woMCI, nor did they correlate with MTL or FS volumes. These findings may relate to the small proportion of PD-woMCI patients who exhibited a cued retrieval-deficit $(14.5 \%)$ or recognition retrieval-deficit ( $8 \%$ ) profile. Indeed, the retrieval hypothesis has been questioned more recently, since the magnitude of impairment in delayed recall and recognition memory is similar in most nondemented PD patients [22-24], as we found in PD-woMCI. In prior studies, the absence of a benefit in recognition memory testing relative to free recall was due to increased false positives on recognition testing [23], presumably owing to FS-based disinhibition. However, recognition memory false positives and cued recall intrusions were not elevated in our PD-woMCI group, nor were these measures related to FS volumes. Altogether, retrieval impairments in PD-woMCI were uncommon, which likely rendered correlations 
between retrieval indices and regional volumes insensitive.

Limitations of our study include the high educational levels of many participants, which may limit the generalizability of the results. Although a more stringent uncorrected statistical threshold of $p \leq 0.01$ was adopted in lieu of overly conservative adjustments, future studies are needed to validate our findings. Additionally, our level 1 criteria for MCI did not include a language assessment, however language is usually intact in PD patients without deficits in other cognitive domains [28]. Level 1 criteria also provide less diagnostic certainty than level 2 criteria [6,31]. Thus, larger studies with comprehensive level 2 PD-MCI diagnosis should be used in future studies to substantiate these findings. Although learning did not correlate with our measures of executive, attention, and visuospatial functioning, it is possible that other aspects of functioning in these domains not measured in this study (e.g., problem solving, cognitive flexibility, visuoconstruction) could be associated with poorer learning in PD-woMCI. Another caveat is that functional changes in the FS system may be more broadly associated with changes in different facets of memory in PDwoMCI than suggested by our results and possibly precede macrostructural changes in grey matter [44]. Indeed, though 18-month increases in gray-matter thinning of the caudal middle-frontal cortex in PD-woMCI patients was recently found, cortical thinning was not associated with performances on global dementia rating scales [45]. Microstructural changes in FS fiber pathways could also affect executive functions that support various components of memory [46], as well as changes in other pathological processes that alter dopaminergic and non-dopaminergic neurotransmitter systems [47]. Nonetheless, structural MRI will remain an important marker of cognitive decline as it is sensitive to changes in cognition and has advantages over other imaging methods, including its reliability and validation for use in clinical settings.

In summary, subtle changes in several different facets of memory in PD-woMCI were predominantly associated with MTL volumes, whereas cued retrieval and recognition memory were associated with FS volumes. This was found despite an absence of MTL and frontal cortex atrophy. Our results build upon emerging evidence linking structural changes in the MTL with memory function in PD-woMCI [7] and risk for future cognitive decline [12]. Longitudinal studies underway will directly determine if these associations are markers of future memory decline, which if substantiated may help establish cut-off values (e.g., smaller MTL and FS volumes combined with poorer memory) that signify a greater risk for memory impairment.

\section{ACKNOWLEDGMENTS}

The authors would like to thank Gabriel Castillo and Jason Reed for their technical support in acquiring and analyzing the structural MRI data, Colleen Takahashi for her technical support in the preparation of figures, Christopher Fong for his assistance in data collection, and Dr. David Song for referring patients to the study. This research was supported by grants from the Department of Veterans Affairs (DLH: CX000146; JVF: CX12004).

\section{CONFLICT OF INTEREST}

The authors have no conflict of interest to report.

\section{REFERENCES}

[1] Aarsland D, Andersen K, Larsen JP, Lolk A, \& KraghSorensen P (2003) Prevalence and characteristics of dementia in Parkinson disease: An 8-year prospective study. Arch Neurol, 60, 387-392.

[2] Aarsland D, Larsen JP, Tandberg E, \& Laake K (2000) Predictors of nursing home placement in Parkinson's disease: A population-based, prospective study. J Am Geriatr Soc, 48, 938-942.

[3] Hughes TA, Ross HF, Mindham RH, \& Spokes EG (2004) Mortality in Parkinson's disease and its association with dementia and depression. Acta Neurol Scand, 110, 118-123.

[4] Broeders M, Velseboer DC, de Bie R, Speelman JD, Muslimovic D, Post B, de HR, \& Schmand B (2013) Cognitive change in newly-diagnosed patients with Parkinson's disease: A 5-year follow-up study. J Int Neuropsychol Soc, 19, 695708

[5] Muslimovic D, Post B, Speelman JD, \& Schmand B (2005) Cognitive profile of patients with newly diagnosed Parkinson disease. Neurology, 65, 1239-1245.

[6] Litvan I, Goldman JG, Troster AI, Schmand BA, Weintraub D, Petersen RC, Mollenhauer B, Adler CH, Marder K, WilliamsGray CH, Aarsland D, Kulisevsky J, Rodriguez-Oroz MC, Burn DJ, Barker RA, \& Emre M (2012) Diagnostic criteria for mild cognitive impairment in Parkinson's disease: Movement Disorder Society Task Force guidelines. Mov Disord, 27, 349356.

[7] Beyer MK, Bronnick KS, Hwang KS, Bergsland N, Tysnes OB, Larsen JP, Thompson PM, Somme JH, \& Apostolova LG (2013) Verbal memory is associated with structural hippocampal changes in newly diagnosed Parkinson's disease. $J$ Neurol Neurosurg Psychiatry, 84, 23-28.

[8] Weintraub D, Doshi J, Koka D, Davatzikos C, Siderowf AD, Duda JE, Wolk DA, Moberg PJ, Xie SX, \& Clark CM (2011) Neurodegeneration across stages of cognitive decline in Parkinson disease. Arch Neurol, 68, 1562-1568. 
[9] Ibarretxe-Bilbao N, Ramirez-Ruiz B, Tolosa E, Marti MJ, Valldeoriola F, Bargallo N, \& Junque C (2008) Hippocampal head atrophy predominance in Parkinson's disease with hallucinations and with dementia. J Neurol, 255, 1324-1331.

[10] Jokinen P, Bruck A, Aalto S, Forsback S, Parkkola R, \& Rinne JO (2009) Impaired cognitive performance in Parkinson's disease is related to caudate dopaminergic hypofunction and hippocampal atrophy. Parkinsonism Relat Disord, 15, 88-93.

[11] Bruck A, Kurki T, Kaasinen V, Vahlberg T, \& Rinne JO (2004) Hippocampal and prefrontal atrophy in patients with early non-demented Parkinson's disease is related to cognitive impairment. J Neurol Neurosurg Psychiatry, 75, 1467-1469.

[12] Goldman JG, Stebbins GT, Bernard B, Stoub TR, Goetz CG, \& Detoledo-Morrell L (2012) Entorhinal cortex atrophy differentiates Parkinson's disease patients with and without dementia. Mov Disord, 27, 727-734.

[13] Bouchard TP, Malykhin N, Martin WR, Hanstock CC, Emery DJ, Fisher NJ, \& Camicioli RM (2008) Age and dementiaassociated atrophy predominates in the hippocampal head and amygdala in Parkinson's disease. Neurobiol Aging, 29, 10271039.

[14] Davidson PS, Troyer AK, \& Moscovitch M (2006) Frontal lobe contributions to recognition and recall: Linking basic research with clinical evaluation and remediation. J Int Neuropsychol Soc, 12, 210-223.

[15] Rugg MD, Otten LJ, \& Henson RN (2002) The neural basis of episodic memory: Evidence from functional neuroimaging. Philos Trans R Soc Lond B Biol Sci, 357, 1097-1110.

[16] Filoteo JV, Reed JD, Litvan I, \& Harrington DL (2014) Volumetric correlates of cognitive functioning in nondemented patients with Parkinson's disease. Mov Disord, 29, 360-367.

[17] Massman PJ, Delis DC, Butters N, Levin BE, \& Salmon DP (1990) Are all subcortical dementias alike? Verbal learning and memory in Parkinson's and Huntington's disease patients. $J$ Clin Exp Neuropsychol, 12, 729-744.

[18] Pillon B, Deweer B, Agid Y, \& Dubois B (1993) Explicit memory in Alzheimer's, Huntington's, and Parkinson's diseases. Arch Neurol, 50, 374-379.

[19] Flowers KA, Pearce I, \& Pearce JM (1984) Recognition memory in Parkinson's disease. JNeurol Neurosurg Psychiatry, 47, 1174-1181.

[20] Taylor AE, Saint-Cyr JA, \& Lang AE (1990) Memory and learning in early Parkinson's disease: Evidence for a "frontal lobe syndrome“. Brain Cogn, 13, 211-232.

[21] Bondi MW, Kaszniak AW, Bayles K, \& Vance KT (1993) The contributions of frontal system dysfunction to memory and perceptual abilities in Parkinson's disease. Neuropsychol, 7, 89-102.

[22] Whittington CJ, Podd J, \& Kan MM (2000) Recognition memory impairment in Parkinson's disease: Power and metaanalyses. Neuropsychol, 14, 233-246.

[23] Zizak VS, Filoteo JV, Possin KL, Lucas JA, Rilling LM, Davis JD, Peavy G, Wong A, \& Salmon DP (2005) The ubiquity of memory retrieval deficits in patients with frontal-striatal dysfunction. Cogn Behav Neurol, 18, 198-205.

[24] Higginson CI, Wheelock VL, Carroll KE, \& Sigvardt KA (2005) Recognition memory in Parkinson's disease with and without dementia: Evidence inconsistent with the retrieval deficit hypothesis. J Clin Exp Neuropsychol, 27, 516-528.

[25] Bronnick K, Alves G, Aarsland D, Tysnes OB, \& Larsen JP (2011) Verbal memory in drug-naive, newly diagnosed Parkinson's disease. The retrieval deficit hypothesis revisited. Neuropsychol, 25, 114-124.
[26] Weintraub D, Moberg PJ, Culbertson WC, Duda JE, \& Stern MB (2004) Evidence for impaired encoding and retrieval memory profiles in Parkinson disease. Cogn Behav Neurol, 17, 195-200.

[27] Delis DC, Kramer JH, Kaplan E, Ober BA. (2000) California Verbal Learning Test, 2nd ed, The Psychological Corporation, San Antonio.

[28] Loftus AM, Bucks RS, Thomas M, Kane R, Timms C, Barker RA, \& Gasson N (2015) Retrospective assessment of movement disorder society criteria for mild cognitive impairment in Parkinson's disease. J Int Neuropsychol Soc, 21, 137-145.

[29] Szeto JY, Mowszowski L, Gilat M, Walton CC, Naismith SL, \& Lewis SJ (2015) Assessing the utility of the Movement Disorder Society Task Force Level 1 diagnostic criteria for mild cognitive impairment in Parkinson's disease. Parkinsonism Relat Disord, 21, 31-35.

[30] Hobson P, \& Meara J (2015) Mild cognitive impairment in Parkinson's disease and its progression onto dementia: A 16year outcome evaluation of the Denbighshire cohort. Int $J$ Geriatr Psychiatry, 30, 1048-1055.

[31] Goldman JG, Holden S, Ouyang B, Bernard B, Goetz CG, \& Stebbins GT (2015) Diagnosing PD-MCI by MDS task force criteria: How many and which neuropsychological tests? Mov Disord, 30, 402-406.

[32] Filoteo JV, Rilling LM, Cole B, Williams BJ, Davis JD, \& Roberts JW (1997) Variable memory profiles in Parkinson's disease. J Clin Exp Neuropsychol, 19, 878-888.

[33] Desikan RS, Segonne F, Fischl B, Quinn BT, Dickerson BC, Blacker D, Buckner RL, Dale AM, Maguire RP, Hyman BT, Albert MS, \& Killiany RJ (2006) An automated labeling system for subdividing the human cerebral cortex on MRI scans into gyral based regions of interest. Neuroimage, 31, 968-980.

[34] Perneger TV (1998) What's wrong with Bonferroni adjustments. BMJ, 316, 1236-1238.

[35] Shohamy D, \& Adcock RA (2010) Dopamine and adaptive memory. Trends Cogn Sci, 14, 464-472.

[36] Petrou M, Bohnen NI, Muller ML, Koeppe RA, Albin RL, \& Frey KA (2012) Abeta-amyloid deposition in patients with Parkinson disease at risk for development of dementia. Neurology, 79, 1161-1167.

[37] Bohnen NI, Muller ML, Kotagal V, Koeppe RA, Kilbourn MR, Gilman S, Albin RL, \& Frey KA (2012) Heterogeneity of cholinergic denervation in Parkinson's disease without dementia. J Cereb Blood Flow Metab, 32, 1609-1617.

[38] Corbetta M, Patel G, \& Shulman GL (2008) The reorienting system of the human brain: From environment to theory of mind. Neuron, 58, 306-324.

[39] Corbetta M, \& Shulman GL (2002) Control of goal-directed and stimulus-driven attention in the brain. Nat Rev Neurosci, 3, 201-215.

[40] Shulman GL, McAvoy MP, Cowan MC, Astafiev SV, Tansy AP, d'Avossa G, \& Corbetta M (2003) Quantitative analysis of attention and detection signals during visual search. $J$ Neurophysiol, 90, 3384-3397.

[41] Diana RA, Yonelinas AP, \& Ranganath C (2007) Imaging recollection and familiarity in the medial temporal lobe: A three-component model. Trends Cogn Sci, 11, 379-386.

[42] Ward AM, Schultz AP, Huijbers W, van Dijk KR, Hedden T, \& Sperling RA (2014) The parahippocampal gyrus links the default-mode cortical network with the medial temporal lobe memory system. Hum Brain Mapp, 35, 1061-1073. 
[43] Yassa MA, \& Stark CE (2008) Multiple signals of recognition memory in the medial temporal lobe. Hippocampus, 18, 945954.

[44] Harrington DL, Castillo GN, Reed JD, Song DD, Litvan I, \& Lee RR (2014) Dissociation of Neural Mechanisms for Intersensory Timing Deficits in Parkinson's Disease. Timing \& Time Perception, 2, 145-168.

[45] Mak E, Su L, Williams GB, Firbank MJ, Lawson RA, Yarnall AJ, Duncan GW, Owen AM, Khoo TK, Brooks DJ, Rowe JB, Barker RA, Burn DJ, \& O'Brien JT (2015) Baseline and longitudinal grey matter changes in newly diagnosed Parkinson's disease: ICICLE-PD study. Brain, 138, 2974-2986.

[46] Theilmann RJ, Reed JD, Song DD, Huang MX, Lee RR, Litvan I, \& Harrington DL (2013) White-matter changes correlate with cognitive functioning in Parkinson's disease. Front Neurol, 4, 37.

[47] Gratwicke J, Jahanshahi M, \& Foltynie T (2015) Parkinson's disease dementia: A neural networks perspective. Brain, 138, 1454-1476. 